\title{
Evidence-Based Medicine Research on Shanghan Zabing Lun Prescriptions in the Treatment of Endometriosis
}

\author{
Junsheng Song ${ }^{*}$, Hongyu Liu ${ }^{2}$, Mengmeng Han² \\ ${ }^{1}$ Tianjin University of Traditional Chinese Medicine, Tianjin, China \\ ${ }^{2}$ Graduate School, Tianjin University of Traditional Chinese Medicine, Tianjin, China \\ Email: ^songjunsheng00@126.com
}

How to cite this paper: Song, J.S., Liu, H.Y. and Han, M.M. (2016) Evidence-Based Medicine Research on Shanghan Zabing Lun Prescriptions in the Treatment of Endometriosis. Open Journal of Obstetrics and Gynecology, 6, 769-784.

http://dx.doi.org/10.4236/ojog.2016.612095

Received: October 13, 2016

Accepted: November 27, 2016

Published: November 30, 2016

Copyright $\odot 2016$ by authors and Scientific Research Publishing Inc. This work is licensed under the Creative Commons Attribution International License (CC BY 4.0).

http://creativecommons.org/licenses/by/4.0/

\begin{abstract}
Objective: Adopting the methods of Evidence-based Medicine research to acquire the dominant prescriptions of Shanghan Zabing Lun in the treatment of Endometriosis, meanwhile to fully dig the value of classical prescriptions. Method: Firstly, we searched and collected the literatures from last three decades in CNKI, Wanfang and VIP databases, which are about classical prescriptions treating endometriosis. Then, by screening all the literatures, we obtained the clinical research literatures and individual case reports. Finally, we went through the internal quality of the two categories of literatures to get the dominant prescriptions. Results: 22 Shanghan Zabing Lun prescriptions have been used in the treatment of endometriosis. According to the clinical research and case literatures, Guizhi Fuling Wan is the prime prescription to treat endometriosis with high quality and high frequency. Conclusions: Through evidence-based medical research and evaluation of evidence, it can be seen that treatment of endometriosis presents a certain tendency that Guizhi Fuling Wan, Wenjing Decoction and Dahuang Zhechong Wan have good clinical effect treating endometriosis and their syndromes are well matched with the symptoms of endometriosis in the present clinical environment.
\end{abstract}

\section{Keywords}

Shanghan Zabing Lun, Endometriosis, Evidence-Based Medicine, Guizhi Fuling Wan

\section{Introduction}

Endometriosis is one of the most common gynecological diseases, affecting many women's lives and many women were troubled by it [1]. Understanding the causes is essential to treat endometriosis. One of the reasons for endometriosis is congenital re- 
productive organs dysplasia, including uterus atresia, transverse vaginal septum, atresia hymenalis and malposition of the uterus. Also, this can be acquired from abortions, uterus or vaginal surgeries, vaginal drug use, which can result in adhesions of cervix, vagina and introitus. Because of the above pathological states, menstrual blood cannot be eliminated from the body, leading to menstrual blood refluxing into the pelvic cavity.

The treatments in western medicine commonly apply minimally invasive surgeries or medication, while Chinese medicine has good curative effect for endometriosis except caused by congenital reproductive organs dysplasia. By applying evidence-based medicine retrospective study from 1979 to 2013, the following formulas have good curative effect for the disease. Endometriosis belongs to the range of dysmenorrhea, infertility, menstrual disorders and abdominal mass in Traditional Chinese Medicine, which are more often the results of the imbalance between the Chong and Ren Meridians, qi and blood stasis, long-term menstrual abnormalities caused by emotion damage, diet disorders, and unhealthy daily habits.

\section{Prescription Table}

In Shanghan Zabing Lun (Treatise on Febrile and Miscellaneous Diseases), a total of 22 prescriptions are able to treat endometriosis, 13 of which have been gone through clinical studies and 16 of which have been reported in cases experience. Research shows that as of 2013, there have been 84 clinical research literatures and 52 clinical cases literatures published. Among clinical researches, 39 of them are randomized controlled trials, 5 of them are half randomized controlled trials, 11 of them are controlled trials and 29 of them are cases series observations. Clinical cases literatures report 54 cases in total. The prescriptions with high frequency are shown in Table 1 and Table 2, demonstrating Guizhi Fuling Wan, Dahuang Zhechong Wan, Didang Decoction, Wenjing Decoction and Xiayuxue Decoction are more applied in cinical research literature and Guizhi Fuling Wan, Wenjing Decoction, Sini Powder and Danggui Shaoyao Powder are more applied in clinical cases.

\section{Prescriptions and Quality Level in Clinical Research Literatures}

Evidence-based medicine commonly refer to GRADE standard to evaluate quality of literatures, whereas Evidence-based medicine research in Traditional Chinese Medicine cannot ponderously apply the exact same items. Consequently, the following quality

Table 1. Frequent prescriptions in clinical research literature.

\begin{tabular}{ccc}
\hline Serial Number & Prescription & Frequency \\
\hline 1 & Guizhi Fuling Wan & 60 \\
2 & Dahuang Zhechong Wan & 6 \\
3 & Didang Decoction & 4 \\
4 & Wenjing Decoction & 3 \\
5 & Xiayuxue Decoction & 3 \\
\hline
\end{tabular}


Table 2. Frequent prescriptions in clinical cases literature.

\begin{tabular}{ccc}
\hline Serial Number & Prescription & Frequency \\
\hline 1 & Guizhi Fuling Wan & 22 \\
2 & Wenjing Decoction & 5 \\
3 & Sini Powder & 5 \\
4 & Danggui Shaoyao Powder & 4 \\
\hline
\end{tabular}

level identification is based on a modified GRADE standard that was discussed and recognized by several rounds of professionals, adding four items of the standard which are: addition or subtraction of prescriptions, the original does and composition of the prescription, using Shanghan Zabing Lun prescriptions only and whether researches and cases are from before the year of 1979. The rest of items are the same as in GRADE standard [2]. Detailed information is shown in Table 3.

\subsection{Guizhi Fuling Wan}

59 papers are included in total, 28 of which are randomized controlled trials, 5 are half randomized controlled trials, 8 are controlled trials, and 18 cases series observations. All the documents were published between 1992-2013.

Quality grade evaluation of evidence is shown in Table 4. It shows that there are 14 pieces of high $(\mathrm{H})$ quality evidence, 17 of medium (M) quality, 12 of low (L) quality, 16 of very low (VL) quality. Evidence quality level degrading factors mainly are the research limitations (1), addition or subtraction of prescriptions (2), low accuracy (3) and indirect evidence (4). Evidence quality level upgrading factors mainly are using the original prescription of Shanghan Zabing Lun (5), or only using traditional Chinese medicinal intervention (6).

\subsection{Dahuang Zhechong Wan}

6 papers are included in total, 2 of which are randomized controlled trials, 1 is controlled trials, and 3 cases series observations. All the documents were published between 1998-2001. Quality grade evaluation of evidence is shown in Table 5. It shows that

Table 3. Deciding factors in quality level.

\begin{tabular}{cc}
\hline Influence of Orientation & Specific Factors \\
\hline Degrading Factors & Research Limitation \\
Inconsistent Results \\
Indirect Evidence \\
Low Accuracy \\
Publication Bias \\
Upgrading Factors & Addition or Subtraction of Prescriptions \\
& Original Does \& Composition of the Prescription \\
Using Shanghan Zabing Lun Prescriptions Only \\
Researches and Cases from Before Year of 1979 \\
High Effective Value \\
Miscellaneous factors Reducing Clinical Effect \\
Does-Effect Relationship
\end{tabular}


there are 1 piece of high $(\mathrm{H})$ quality evidence, 3 of medium (M) quality, 2 of low (L) quality, 16 of very low (VL) quality. Evidence quality level degrading factors mainly are the research limitations (1) and low accuracy (3). Evidence quality level upgrading factors mainly are using the original prescription of Shanghan Zabing Lun (5), or only using traditional Chinese medicinal intervention (6).

\subsection{Didang Decoction}

4 papers are included in total, 2 of which are randomized controlled trials and 2 cases series observations. All the documents were published between 2003-2012. Quality

Table 4. Quality level in clinical research literatures of Guizhi Fuling Wan.

\begin{tabular}{|c|c|c|c|c|}
\hline Research & Pulish Year & Type & Factors & Level \\
\hline Yang Hong [3] & 2013 & RCT & (5) $(+1)$ & $\mathrm{H}$ \\
\hline Gu Ziyang [4] & 2012 & RCT & (1) $(-1)$ (6) $(+1)$ & $\mathrm{H}$ \\
\hline $\begin{array}{c}\text { Xu Jie-Huo Xiangyun } \\
{[5]-[16]}\end{array}$ & $2007-2013$ & RCT-CR & (1) $(0,-1,-2)(5)(+1)(6)(+1)$ & $\mathrm{H}$ \\
\hline Wang Liying [17] & 2013 & RCT & (1) $(-2)(5)(+1)$ & M \\
\hline Sun Hui $[18]$ & 2012 & CT & (1) $(-2)(5)(+1)$ & M \\
\hline Zhang Cuirong [19] & 2011 & $\mathrm{CR}$ & (5) $(+1)$ & M \\
\hline $\begin{array}{l}\text { Wang Chong-Zheng } \\
\text { Xiujuan [20]-[33] }\end{array}$ & $1995-2013$ & RCT-CR & $\begin{array}{c}\text { (1) }(0,-1,-2) \text { (3) }(-1)(5)(+1) \text { (6) } \\
(+1)\end{array}$ & $\mathrm{M}$ \\
\hline Huang Xunfang [34] & 2013 & RCT & (1) $(-2)(3)(-1)(5)(+1)$ & $\mathrm{L}$ \\
\hline $\begin{array}{c}\text { Yang } \\
\text { Yaqin [35] }\end{array}$ & 2013 & $\mathrm{CT}$ & (1) $(-2)(3)(-1)(5)(+1)$ & $\mathrm{L}$ \\
\hline Zhang Lifan [36] & 2004 & $\mathrm{CCT}$ & (1) $(-1)(3)(-1)(2)(-1)$ (6) $(+1)$ & $\mathrm{L}$ \\
\hline $\begin{array}{l}\text { Wang Ye-Zhong Ruifang } \\
\text { [37]-[45] }\end{array}$ & $2000-2011$ & $\begin{array}{c}\mathrm{RCT}, \\
\mathrm{CR}\end{array}$ & 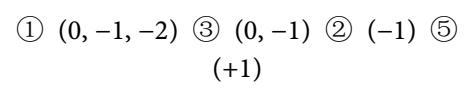 & $\mathrm{L}$ \\
\hline Li Yili [46] & 2013 & RCT & (1) $(-1)(4)(-1)(3)(-1)(2)(-1)(5)(+1)$ & $\mathrm{VL}$ \\
\hline Li Aifang [47] & 2012 & $\mathrm{CT}$ & (1) $(-2)(2)(-1)$ & $\mathrm{VL}$ \\
\hline $\begin{array}{l}\text { Hu Fang-Gao Huiming } \\
{[48]-[61]}\end{array}$ & $1992-2012$ & $\begin{array}{c}\mathrm{RCT}, \\
\mathrm{CR}\end{array}$ & 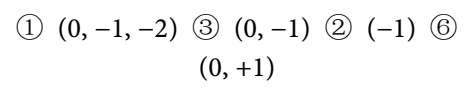 & VL \\
\hline
\end{tabular}

Table 5. Quality level in clinical research literatures of Dahuang Zhechong Wan.

\begin{tabular}{ccccc}
\hline Research & Pulish Year & Type & Factors & Level \\
\hline Li Mingzhou [62] & 2007 & RCT & (1) (-2) (5) (+1) (6) (+1) & H \\
Wei Lijun [63] & 1998 & CR & (1) (-1) (5) (+1) (6) (+1) & M \\
Jin Tianfu [64] & 2005 & CR & (5) (+1) & M \\
Liu XiuFeng [65] & 2004 & CT & (1) (-2) (3) (-1) (5) (+1) (6) (+1) & M \\
Fan Dongxian [66] & 2004 & CR & (1) (-1) (5) (+1) & L \\
Ji Zhaofang [67] & 2011 & RCT & (1) (-2) (3) (-1) (5) (+1) & L \\
\hline
\end{tabular}


grade evaluation of evidence is shown in Table 6. It shows that there are 3 of low (L) quality, 1 of very low (VL) quality. Evidence quality level degrading factors mainly are the research limitations (1) and addition or subtraction of prescriptions (2). Evidence quality level upgrading factors mainly is using traditional Chinese medicinal intervention (6).

\subsection{Wenjing Decoction}

3 papers are included in total, 2 of which are randomized controlled trials and 1 is controlled trials. All the documents were published between 1998-2012. Quality grade evaluation of evidence is shown in Table 7. It shows that there are 1 piece of high $(\mathrm{H})$ quality evidence, 2 of medium (M) quality. Evidence quality level degrading factors mainly is the research limitations (1). Evidence quality level upgrading factors mainly are using the original prescription of Shanghan Zabing Lun (5), or only using traditional Chinese medicinal intervention (6).

\subsection{Xiayuxue Decoction}

3 papers are included in total, 1 of which is randomized controlled trial, 1 is controlled trial, and 1 cases series observation. All the document were published between 20012012. Quality grade evaluation of evidence is shown in Table 8. It shows that there are 1 piece of medium (M) quality evidence, 2 of very low (VL) quality. Evidence quality level degrading factors mainly are the research limitations (1), addition or subtraction of prescriptions (2) and does-effect relationship (7). Evidence quality level upgrading factors mainly is only using traditional Chinese medicinal intervention (6).

\subsection{Prescriptions with Low Frequency}

Another eight prescriptions, all have only 1 evidence support, respectively, Sini Powder, Sini Decoction, Danggui Sini plus Wuzhuyu Shengjiang Decoction, Shenqi Wan,

Table 6. Quality level in clinical research literatures of Didang decoction.

\begin{tabular}{ccccc}
\hline Research & Pulish Year & Type & Factors & Level \\
\hline Wang Zhen [68] & 2009 & CR & (2) (-1) (6) (+1) & L \\
Duan Qingzhen [69] & 2012 & RCT & (1) (-2) (2) (-1) (6) (+1) & L \\
Zeng Jibao [70] & 2008 & RCT & (1) (-2) (2) (-1) (6) (+1) & L \\
Wu Xuehua [71] & 2003 & CR & (1) (-1) (2) (-1) (6) (+1) & VL \\
\hline
\end{tabular}

Table 7. Quality level in clinical research literatures of Wenjing decoction.

\begin{tabular}{ccccc}
\hline Research & $\begin{array}{c}\text { Publish } \\
\text { Year }\end{array}$ & Type & Factors & Level \\
\hline Zhang Yongluo [72] & 1998 & CT & (1) $(-2)(5)(+1)(6)(+1)$ & H \\
Zhen Haiping [73] & 2012 & RCT & (1) (-2) (6) (+1) & M \\
Chen Jianxin [74] & 2003 & RCT & (1) (-2) (5) (+1) & M \\
\hline
\end{tabular}


Table 8. Quality level in clinical research literatures of Xiayuxue decoction.

\begin{tabular}{ccccc}
\hline Research & Publish Year & Type & Factors & Level \\
\hline Li Aifang [75] & 2012 & CT & (1) $(-2)(2)(-1)(7)(+1)(6)(+1)$ & M \\
Zhu Zhenhua [76] & 2001 & CR & (1) $(-1)(2)(-1)(6)(+1)$ & VL \\
Hou Zhixia [77] & 2010 & RCT & (1) (-2) (2) (-1) (6) (+1) & VL \\
\hline
\end{tabular}

Huangqi Guizhi Wuwu Tang, Taohe Chenqi Decoction, Danggui Shaoyao Powder, Biejiajian Wan. Quality grade evaluation of evidence is shown in Table 9. It shows that there are 1 piece of high $(\mathrm{H})$ quality evidence, 1 of low $(\mathrm{L})$ quality, 6 of very low (VL) quality. Evidence quality level degrading factors mainly are the research limitations (1), addition or subtraction of prescriptions (2), low accuracy (3). Evidence level upgrading factors mainly are using the original prescription of Shanghan Zabing Lun (5), or only using traditional Chinese medicinal intervention (6).

\section{Clinical Cases Literature}

54 cases are included in total mainly using Guizhi Fuling Wan, Wenjing Decoction, Sini Powder, Danggui Shaoyao Powder and Danggui Shaoyao Powder. All the documents were published between 1984-2013. Quality grade evaluation of evidence of high frequent prescriptions is shown in Table 10. It shows that cases quality level is mainly medium (M) and low (L).

\section{Typical Clinical Evidence}

There are 83 clinical research literatures in total using prescriptions of Shanghan Zabing Lun treating endometriosis, 17 of which are of high quality, 23 of medium quality, 18 of low quality and 25 of very low quality. High and medium quality evidence mainly comes from Guizhi Fuling Wan related literatures.

Table 9. Quality level in clinical research literatures of low frequent prescriptions.

\begin{tabular}{|c|c|c|c|c|c|}
\hline Research & Prescription & $\begin{array}{l}\text { Publish } \\
\text { Year }\end{array}$ & Type & Factors & Level \\
\hline Zhu Mei [78] & $\begin{array}{c}\text { Danggui Sini plus } \\
\text { Wuzhuyu Shengjiang } \\
\text { Decoction }\end{array}$ & 2012 & RCT & (1) $(-2)(5)(+1)(6)(+1)$ & $\mathrm{H}$ \\
\hline Li Guoxin [79] & Biejiajian Wan & 1997 & CR & (1) $(-1)(2)(-1)(5)(+1)(6)(+1)$ & $\mathrm{L}$ \\
\hline Xue Yufang [80] & Shenqi Wan & 2006 & $\mathrm{RCT}$ & (1) $(-2)(3)(-1)(2)(-1)$ & $\mathrm{VL}$ \\
\hline Liang Qiuxia [81] & $\begin{array}{l}\text { Huangqi Guizhi } \\
\text { Wuwu Tang }\end{array}$ & 2002 & RCT & (1) $(-2)$ (3) $(-1)(2)(-1)(6)(+1)$ & $\mathrm{VL}$ \\
\hline Huang Xirong [82] & $\begin{array}{l}\text { Taohe Chenqi } \\
\text { Decoction }\end{array}$ & 2007 & $\mathrm{CR}$ & (1) $(-1)(2)(-1)(6)(+1)$ & $\mathrm{VL}$ \\
\hline Zheng Meihua [83] & $\begin{array}{c}\text { Danggui Shaoyao } \\
\text { Powder }\end{array}$ & 2001 & $\mathrm{CR}$ & (1) $(-1)(2)(-1)$ (6) $(+1)$ & $\mathrm{VL}$ \\
\hline Wang Shasha $[84]$ & Sini Decoction & 2012 & $\mathrm{RCT}$ & (1) $(-2)$ (3) $(-1)(2)(-1)$ (6) $(+1)$ & $\mathrm{VL}$ \\
\hline Cheng Huilian [85] & Sini Powder & 2009 & CT & (1) $(-2)$ (3) $(-1)(2)(-1)$ (6) $(+1)$ & VL \\
\hline
\end{tabular}


Table 10. Quality level in clinical cases literature.

\begin{tabular}{ccccc}
\hline Prescription & Publish Year & Quantity & Average Quality Score & Level \\
\hline Guizhi Fuling Wan & $1984-2013$ & 22 & 33.65 & $\mathrm{M}$ \\
Wenjing Decoction & $1985-2011$ & 5 & 38.94 & $\mathrm{M}$ \\
Sini Powder & $1995-2012$ & 5 & 44.38 & $\mathrm{M}$ \\
Danggui Shaoyao Powder & $1995-2003$ & 4 & 26.61 & $\mathrm{~L}$ \\
\hline
\end{tabular}

\subsection{Guizhi Fuling Wan}

Guizhi Fuling Wan capsule combined with Mifepristone contrasting mere Mifepristone in the treatment of endometriosis has advantages in clinical total effective rate (high quality evidence).

Pan Xiurong's [6] implementation of a clinical randomized controlled trial, with the sample size of 85 cases: Experimental group 44 cases, control group 41 cases. Control group patients start taking mifepristone (Beijing zizhu pharmaceutical co., LTD., production) on the first day of menstruation, $12.5 \mathrm{mg}$ each time, 1 time/d, lasting for six months. Patients in the experimental group, besides the treatment as control group provides, take Guizhi Fuling Wan capsule (Jiangsu kang pharmaceutical co., LTD.) every time 3 grains $(0.93 \mathrm{~g}), 3$ times/d, lasting for six months. Comparing the two groups, the clinical total effective relative risk (RR) of $1.23,95 \% \mathrm{CI}(1.02,1.02), \mathrm{P}=0.03$, with statistical significance. The curative effect standards: Recovery: Symptoms disappear, pelvic mass or some local symptoms basically disappear. Effective: Symptoms, pelvic mass is not increasing or slightly shrinks. Invalid: Main symptoms are not improving or even getting worse, local lesion is exacerbating. Recurrence: After surgery and drug treatment, or after lesions and symptoms shrinking or disappearing, clinical symptoms restore again to the level before treatment or aggravate.

\subsection{Dahuang Zhechong Wan}

Dahuang Zhechong Wan combined with Gestrinone contrasting mere Gestrinone in the treatment of endometriosis has advantages in clinical total effective rate (high quality evidence).

Li Mingzhou et al.'s [62] implementation of a clinical randomized controlled trial, with a sample size of 120 cases, experimental group of 60 and control group of 60: $\mathrm{Pa}-$ tients take Dahuang Zhechong Wan (Z4202077, produced by Wuhan Zhonglian Pharmaceutical Company), $3 \mathrm{~g}$, 2 times/d after meals, stop taking in menstruation, Gestrinone $25 \mathrm{mg}$ once daily, after meals. The control group merely takes Gestrinone $25 \mathrm{mg}$ once daily, after meals. Comparing the two groups, the clinical total effective relative risk (RR) of $1.11,95 \% \mathrm{CI}(1.01,1.01), \mathrm{P}=0.03$, with statistical significance. The curative effect standards: Recovery: Symptoms disappear, local signs disappeared basically. Prominently effective: Symptoms disappeared basically, mass shrinks more than $1 / 2$ of its size. Effective: Symptoms or physical signs stay in previous condition after treatment (not turning better or worse). 


\subsection{Wenjing Decoction}

WenJing Decoction contrasting Medroxyprogesterone Acetate in the treatment of endometriosis has advantages in clinical total effective rate (high quality evidence).

Zhang Yongluo et al.'s [72] implementation of a controlled clinical trial, with a sample size of 95 cases, experimental group of 45 and control group of 40: Experimental patients take Wenjing Decoction. Composition: Wuzhuyu (Fructus Evodiae) 6 g, Danggui (Radix Angelicae Sinensis) 20 g, Chishao (Radix Paeoniae Rubra) 15 g, Dangshen (Radix Codonopsis) 12 g, Guizhi (Ramulus Cinnamomi) 10 g, Ejiao (Colla Corii Asini) 10 g, Danpi (Cortex Moutan) 10 g, Shengjiang (Rhizoma Zingiberis Recens) 6 g, Gancao (Radix Glycyrrhizae) 6 g, Qingbanxia (Rhizoma Pinelliae) 6 g, Maidong (Radix Ophiopogonis) $6 \mathrm{~g}$, be decocted in water, once/d. 3 months for 1 course of treatment. Patients in control group take Medroxyprogesterone Acetate, from the menstrual cycle day 6th-day 25th, $4 \mathrm{mg}$ each time, 1 time/d, keeping three cycles. Comparing the two groups, the clinical total effective relative risk (RR) of $1.35,95 \% \mathrm{CI}(1.03,1.03), \mathrm{P}=$ 0.03 , with statistical significance. Curative effect standards: Recovery: Symptoms (blood stasis) disappear, pelvic mass or local signs disappear basically, infertility is pregnant in a year. Prominently effective: Symptoms (blood stasis) largely disappear, pelvic mass shrinks (ultrasound contrast in menstrual cycle period before and after the treatment), local signs still exist, but the infertility becomes pregnant within 3 years. Effective: Effectively relieve symptoms, pelvic mass remain unchanged (ultrasound contrast in menstrual cycle period before and after the treatment), conditions do not worsen after 3 months' drug withdrawal. Invalid: Main symptoms do not change or deteriorate, pelvic mass remain unchanged after treatment.

\subsection{Other Prescriptions}

Danggui Sini plus Wuzhuyu Shengjiang Decoction contrasting Duyiwei Capsule in the treatment of endometriosis has advantages in relieving dysmenorrhea (high quality evidence).

Zhu Mei's [78] implementation of a clinical randomized controlled trial, with a sample size of 62, experimental group of 32 and control group of 30: Experimental group patients take Danggui Sini plus Wuzhuyu Shengjiang Decoction: Danggui (Radix Angelicae Sinensis) 15 g, Shaoyao (Radix Paeoniae Alba) 15 g, Zhigancao (Radix Glycyrrhizae) 10 g, Tongcao (Tetrapanax Papyriferus) 10 g, Guizhi (Ramulus Cinnamomi) 10 g, Xixin (Herba Asari) 6 g, Shengjiang (Rhizoma Zingiberis Recens) 10 g, Wuzhuyu (Fructus Evodiae) 6 g, Dazao (Fructus Jujubae) 5, Yellow rice wine $20 \mathrm{ml}$, decocte 1000 $\mathrm{ml}$ water for $300-400 \mathrm{ml}$, take the decoction warmly by 2 times in the morning and evening. Control group: take Duyiwei capsule (Gansu Duyiwei Biological Pharmaceutical co., LTD., 210970053, each grain of $0.3 \mathrm{~g}$ ), 3 capsules per time and 3 times/d. Two groups of comparisons, dysmenorrhea efficacy relative risk (RR) of 1.28, 95\% CI (1.01, 1.01 ), $\mathrm{P}=0.04$, with statistical significance. Curative effect standards: Index: (points before treatment subtracts points after treatment)/points after treatment mutiplies by 100\%. Recovery: Dysmenorrhea symptoms disappear after treatment, integral value de- 
creases $95 \%$ or more. Prominently effective: $95 \%>$ integral value $>70 \%$. Effective: $70 \%>$ integral value $>30 \%$. Invalid: integral decreases $<30 \%$ after treatment.

\section{The Analysis of Dominant Prescriptions Treating Endometriosis}

Endometriosis is a common disease in urogenital system, nowadays, its incidence has been increasing due to rising multipled-pressure in modern society and changed of people eating habits and etc. Based on the principle of TCM syndrome differentiation and treatment, the disease attributes to different disease syndrome. The study found that a total of 25 prescriptions in Shanghan Zabing Lun (Treatise on Febrile and Miscellaneous Diseases) were applied to treat Endometriosis, which is defined as different prescriptions treating same disease. According to the literatures and based on evidencebased medicine study, conclusions are: Guizhi Fuling Wan related papers are a total of 59, including 3648 subjects. Dahuang Zhechong Wan related papers are a total of 6, including 434 subjects. Didang Decoction related is a total of 4 articles, including 287 cases. Wenjing Decoction related is a total of 3 documents, including 201 subjects. Xiayuxue Decoction related is a total of 3 documents, including 191 subjects. Other each prescription related number of literature is one, occupying small percentage. High quality and medium quality clinical evidence are mainly concentrated on Guizhi Fuling Wan. It can be seen that although there are reports of multiple prescriptions treating endometriosis, the high frequency or high quality of evidence literature tend to be aggregating.

\subsection{Guizhi Fuling Wan}

Guizhi Fuling Wan composed by Guizhi (Ramulus Cinnamomi), Fuling (Poria), Danpi (Cortex Moutan), Taoren (Semen Persicae), Baishao (Radix Paeoniae Alba), is from Jinkui Yaolue (Synopsis of Golden Chamber), in woman pregnant disease chapter, treating woman with mass or stasis in the abdomen. Symptoms are abnormal menstruation, amenorrhea and postmenopausal bleeding etc. Endometriosis is a relatively high frequency disease in Guizhi Fuling Wan treating spectrum. There are 14 pieces of high quality evidence and 17 of medium in Guizhi Fuling Wan related papers. 41 controlled clinical trials have shown that combination therapy of Guizhi Fuling Wan and western medicine in the treatment of endometriosis curative effect is superior to the simple application of mifepristone, medroxyprogesterone acetate, levonorgestrel etc. 18 cases of series observations show that Guizhi Fuling Wan and its variations is effective for treating endometriosis. Outcomes are observed mainly on clinical symptoms, B ultrasonic examination, serum CA125 improvement. It can show blood stasis tends to be the pathogenesis of endometriosis in clinical phenomena, which has high degree of aggregation.

\subsection{Dahuang Zhechong Wan}

Dahuang Zhechong Wan composed by Dahuang (Radix et Rhizoma Rhei), Huangqin (Radix Scutellariae), Gancao (Radix Glycyrrhizae), Taoren (Semen Persicae), Xingren

(Semen Armeniacae Amarae), Shaoyao (Radix Paeoniae Alba), GanDihuang (Radix 
Rehmanniae), Ganqi (Resina Toxicodendri), Mangchong (Gadfly), Shuizhi (Hirudo), Qicao (Larva Holotrichiae) and Zhechong (Woodlouse), is from Jinkui Yaolue (Synopsis of Golden Chamber), in Blood Bi and consumptive disease chapter. Endometriosis is a relatively high frequency disease in Dahuang Zhechong Wan treating spectrum. There are 1 piece of high quality evidence, 3 of medium quality and 2 of low quality reports in Dahuang Zhechong Wan related papers. Three controlled clinical trials have shown that Dahuang Zhechong Wan combined with western medicine treating endometriosis is superior to the simple application of gestrinone or danazol etc. Three cases series observations show that Dahuang Zhechong Wan and its variations is effective for treating endometriosis. Outcomes are observed mainly on the improvement in clinical symptoms. It indicates that fatigue illness with blood stasis is the common pathogenesis of endometriosis, although limited evidence and low quality reports, it still can be reference to use coming to the certain disease syndrome.

\subsection{Wenjing Decoction}

WenJing Decoction composed by Wuzhuyu (Fructus Evodiae), Danggui (Radix Angelicae Sinensis), Xiongqiong (Rhizoma Chuanxiong), Shaoyao (Radix Paeoniae Alba), Renshen (Ginseng), Guizhi (Ramulus Cinnamomi), Ejiao (Colla Corii Asini), MudanPi (Cortex Moutan), Shengjiang (Rhizoma Zingiberis Recens), Gancao (Radix Glycyrrhizae), Banxia (Rhizoma Pinelliae) and Maidong (Radix Ophiopogonis), is from Jinkui Yaolue (Synopsis of Golden Chamber), in women miscellaneous diseases chapter. Endometriosis is a relatively low frequency disease in Wenjing Decoction treating spectrum. There are 1 piece of high quality evidence, 2 of medium quality reports in Wenjing Decoction related papers. Three controlled clinical trials have shown that combination therapy of WenJing Decoction and western medicine treating endometriosis is superior to the simple application of gestrinone, medroxyprogesterone acetate, mifepristone and etc. Outcomes are observed mainly on the improvement in clinical symptoms, the improvement of the ultrasound examination. Although the blood stasis is a common pathogenesis of the disease, coldness in body is also a main pathogenesis. Although the evidence is limited, this card type is used for reference. It still can be reference to use coming to the certain disease syndrome.

\subsection{Other Prescriptions}

There is less documentary evidence or low quality or less frequency for other prescriptions, such as Danggui SiNi plus Wuzhuyu Shengjiang Decoction, Shenqi Wan, Huangqi Guizhi Wuwu Decoction, Taohe Chengqi Decoction, Danggui Shaoyao Powder, Biejiajian Wan and etc. The pathogenesises the above prescriptions' syndromes stand for lack of enough evidence to support, but still have curative effect on the disease clinically, they can be used to refer to. More strong evidence from more researchers in the future is expected to improve the curative effect of the disease.

\section{Conclusions}

According to the analysis of existing literature evaluation, it shows that clinical types of 
endometriosis are: Guizhi Fuling Wan syndrome and Didang Decoction syndrome standing for the pathogenesis of stagnation of blood stasis, Dahuang Zhechong Wan syndrome standing for pathogenesis of consumptive internal injuries and blood stasis, Wenjing Decoction syndrome standing for pathogenesis of Chong-Ren coldness and stagnation of blood stasis, Xiayuxue Decoction syndrome standing for pathogenesis of heat and blood stasis.

By evidence-based medicine research and evaluation of evidence, extracting endometriosis treated with prescriptions from Shanghan Zabing Lun presents certain trends. And prescriptions above are likely to be the main syndrome types in endometriosis in current clinical situation (Figure 1).

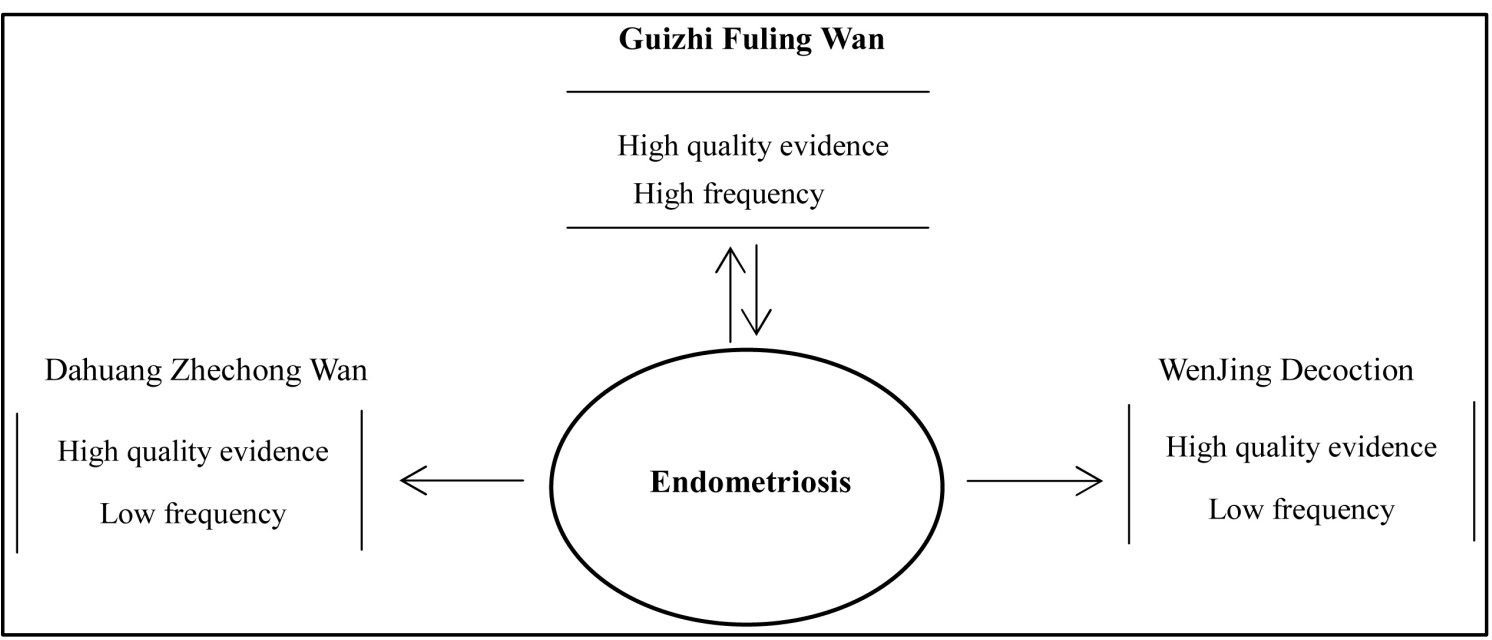

Figure 1. Laws of different endometriosis syndromes.

\section{Acknowledgements}

The Project was supported by the National Natural Science Fund Project: Establish Shanghan Zabing Lun Prescriptions Treating Chronic Diseases Evidence-based Decisionmaking Research Based on Multi-level Evidence Fusion Theory (Number: 81373891).

\section{References}

[1] Lang, J.H. (2009) Recognition and Significance of Endometriosis. Engineering Sciences, 11, 137-138.

[2] Song, J.S. (2012) Research on Laws of Shang Han Lun Prescriptions Treating Frequent Diseases and Syndromes. China Press of Traditional Chinese Medicine, Beijing, 13-14.

[3] Hong, Y. (2013) Joint Curative Effect Observation of Guizhi Fuling Capsule, Mifepristone and Gestrinone Treating Endometriosis. Chinese Journal of Primary Medicine and Pharmacy, 20, 1680-1681.

[4] Gu, Z.Y. (2012) Joint Curative Effect Observation of Guizhi Fuling Capsule and Mifepristone Treating Endometriosis. Strait Pharmaceutical Journal, 24, 193-195.

[5] Xu, J. (2012) Curative Effect Observation of Guizhi Fuling Wan Treating Adenomyosis. Medical Frontier, 2, 389.

[6] Pan, X.R. (2011) Clinical Observation of Mifepristone Combined with Guizhi Fuling Cap- 
sule Treating Endometriosis. China Practical Medicine, 6, 136-137.

[7] Liang, R.L. and Luo, S. (2011) Curative Effect Observation of Levonorgestrel Intrauterine Release System Combined with Guizhi Fuling Capsule Treating Adenomyosis. West China Medical Journal, 26, 72.

[8] Guo, J.L. (2011) Curative Effect Observation of Treatment on 34 Cases of Adenomyosi by Mifepristone Combined with Guizhi Fuling Capsule. Chongqing Medicine, 40, 673.

[9] Chang, G.Z. (2011) Guizhi Fuling Capsule in Treatment of Endometriosis Dysmenorrhea, and Its Influence on Serum CA125. Journal of Emergency in Traditional Chinese Medicine, 20, 831-832.

[10] Li, L.J. and Ma, W.J. (2011) Levonorgestrel Intrauterine Release System Combined with Guizhi Fuling Capsule Treating 50 Caese of Adenomyosis. Chinese Journal of Reproductive Health, 22, 304-305.

[11] Li, Y.J. (2009) Clinical Research of Radiofrequency Ablation Combined with Chinese Medicine Treatment of Adenomyosis. Maternal and Child Health Care of China, 24, 995-996.

[12] Liu, Y. (2009) Mifepristone Combined with Guizhi Fuling Capsule Treating 30 Cases of Adenomyosis. Journal of Aerospace Medicine, 20, 92-97.

[13] Lu, C.H. (2007) Curative Effect Observation of Treatment on Endometriosis by Mifepristone Combined with Guizhi Fuling Capsule. Modern Medicine Journal of China, 9, 50-51.

[14] Shen, W.L. (2013) Curative Effect Observation of Treatment on Endometriosis by Mifepristone Combined with Guizhi Fuling Capsule and Influence on Serum Sex Hormones. Clinical Journal of Chinese Medicine, 5, 28-29.

[15] Liu, Y.C. (2013) Curative Effect Observation of Mifepristone Combined with Guizhi Fuling Capsule Treating Endometriosis Cyst Ultrasound Interventional Surgery. China Journal of Modern Medicine, 23, 92-94.

[16] Huo, Y.X. (2008) Clinical Observation Guizhi Fuling Capsule in the Treatment of Endometriosis and Effects of CA125 and CA19-9. Guiding Journal of Traditional Chinese Medicine and Pharmacy, 14, 54-55.

[17] Wang, L.Y. and Wang, L.P. (2013) Treatment of 30 Cases of Endometriosis by Guizhi Fuling Capsule and Small Dose of Mifepristone. Shaanxi Journal of Traditional Chinese Medicine, 34, 774-775.

[18] Sun, H. (2012) Uterine Adenomyoma Conservative and Surgical Treatment Effect Comparison and Analysis. Modern Preventive Medicine, 39, 3234-3235, 3238.

[19] Zhang, C.R., Ma, S.T., Zhang, Y.H., et al. (2011) Treatment of 35 Cases of Adenomyosis by Guizhi Fuling Capsule and Mifepristone. Hebei Journal of Traditional Chinese Medicine, 33, 1053.

[20] Wang, C. and Shi, Y.L. (2011) Treatment of 90 Cases of Endometriosis by Combining Traditional Chinese and Western Medicine. Journal of Practical Traditional Chinese Internal Medicine, 25, 92-93.

[21] Liu, Y.L., Wang, J.L., Teng, H., et al. (2010) Curative Effect Observation of Guizhi Fuling Capsule Treating 48 Cases of Endometriosis. Shandong Medical Journal, 50, 78.

[22] Lin, T.Y. (2010) Curative Effect Observation of Guizhi Fuling Capsule Combined with Mifepristone Treating Endometriosis. Hebei Medicine, 16, 578-560.

[23] Li, H.Y. (2010) Curative Effect Observation of Guizhi Fuling Wan Treating 73 Cases of Endometriosis. Journal of Community Medicine, 8, 51.

[24] Jiang, Z.Y. (2010) Curative Effect Observation of Guizhi Fuling Wan Combined with Electrochemical Method Treating Endometriosis. China’s Traditional Chinese Medicine Infor- 
mation, 2, 224.

[25] Pang, F.F. (2009) Curative Effect Observation of Combined Traditional Chinese with Western Medicine Preventing and Treating the Post-Operation Relapse of Endometriosis. Shandong Journal of Traditional Chinese Medicine, 28, 413-414.

[26] Yu, J.W., Zhao, L., Li, R.L., et al. (2010) Clinical Observation of Promoting Blood Circulation and Removing Blood Stasis Chinese Herbs Treating Endometriosis. Chinese Journal of Traditional Medical Science and Technology, 17, 344-345.

[27] Chen, J.Y. (2006) Guizhi Fuling Capsule Combined with Medroxyprogesterone Treating Endometriosis. Modern Journal of Integrated Traditional Chinese and Western Medicine, 15,878 .

[28] Yang, X. and Li, S.Y. (2006) Clinical Observation of Guizhi Fuling Capsule and Ba Zhen Yi $\mathrm{Mu}$ Wan Treating 56 Cases of Endometriosis. The Chinese and Western Medical Journal, 4, 52-53.

[29] Zhu, J.A. (2005) Guizhi Fuling Capsule Treating 56 Cases of Adenomyosis. The Chinese Modern Journal of Traditional Chinese and Western Medicine, 3, 1218-1219.

[30] Li, X.F. (2013) Combined Traditional Chinese and Western Medicine Treating 39 Cases of Adenomyosis. Health Horizon, 21, 422-423.

[31] Yang, F.Y. (2011) Effects of CRP and VEGF in Serum with Guizhi Fuling Capsule Treating Adenomyosis Dysmenorrhea Patients. Shandong Journal of Traditional Chinese Medicine, 30, 784-785.

[32] Qin, J.W. and He, Q. (2009) Clinical Observation of Guizhi Fuling Capsule Treating Gynecological Diseases. Guide of China Medicine, 7, 87-88.

[33] Zheng, X.J., Zhou, Y.B. and Hu, M.X. (2007) B Ultrasound Guided Puncture with Mifepristone and Guizhi Fuling Capsule Treating 57 Cases of Ovarian Chocolate Cyst. Zhejiang Clinical Medicine, 9, 624.

[34] Huang, X.F. (2013) Curative Effect Observation of Guizhi Fuling Capsule in Treatment of Endometriosis. Contemporary Medicine, 19, 96.

[35] Yang, Y.Q. (2013) Changes and Significance of Serum Interleukin 2, Superoxide Dismutase, Vascular Endothelial Growth Factor and Carbohydrate Antigen 125 in Endometriosis Patients Before and after Treatment. Clinical Medicine of China, 29, 426-428.

[36] Zhang, L.F., Zhu, Y.D. and Yan, C.P. (2004) Clinical Observation on Treatment of Endometriosis by Guizhi Fuling Capsule and Shen Qi Pill. Chinese Journal of Integrated Traditional and Western Medicine, 24, 859-860.

[37] Wang, Y. (2009) Clinical Observation of Guizhi Fuling Capsule with Short-Acting Contraceptive Treating 34 Cases of Endometriosis. Shanxi Medical Journal, 38, 76-77.

[38] Guo, J.X. (2011) Endometriosis Clinical Research. China Higher Medical Education, No. 4, 141-142.

[39] Gao, N.Q. (2011) Clinical Research of Guizhi Fuling Capsule Combined with Aedroxyprogesterone Acetate Treating Endometriosis. Strait Pharmaceutical Journal, 23, 97-98.

[40] Shen, J.M. (2010) Clinical Observation of Guizhi Fuling Capsule Treating Endometriosis. Chinese Journal of Coal Industry Medicine, 13, 127.

[41] Zhang, H.Z. (2010) The Method of Benefiting Qi and Removing Blood Stasis Treating 38 Cases of Endometriosis. Guangming Traditional Chinese Medicine, 25, 1633-1634.

[42] Xu, J.H. and Zhang, X.D. (2008) Modified Guizhi Fuling Wan Treating 50 Cases of Endometriosis. Henan Traditional Chinese Medicine, 28, 21-22.

[43] Qian, W.C. (2005) Guizhi Fuling Capsule Treating 35 Cases of Endometriosis. Zhejiang 
Journal of Traditional Chinese Medicine, 4, 506.

[44] Jing, Q. (2000) Clinical Research on Guizhi Fuling Capsule Treating Endometriosis. Liaoning Journal of Traditional Chinese Medicine, 24, 170.

[45] Zhong, R.F. (2011) Ultrasonic Intervention with Drug Clinical Analysis for Treatment of Endometriosis Cysts. Image and Intervention, 18, 73-74.

[46] Li, Y.L. (2013) Curative Effect Analysis of Treatment of Endometriosis Cysts by Laparoscopic Surgery Assisting Drug Therapy. Guide of China Medicine, 11, 586-587.

[47] Li, A.F. (2012) Modified Guizhi Fuling Wan and Si Ni Powder Treating Adenomyosis 34 Cases. Clinical Journal of Traditional Chinese Medicine, 24, 676.

[48] Hu, F., You, L. and You, J.Y. (2012) Clinical Observation of Internal and External Treatment of 60 cases Adenomyosis. Yunnan Journal of Traditional Chinese Medicine and Materia Medica, 33, 35-36.

[49] Guo, Y. and Liao, Y. (2010) Recent Clinical Observation of Modified Guizhi Fuling Wan Treating Adenomyosis. Chinese Journal of Traditional Medical Science and Technology, 17, 348-349.

[50] Tao, P.M. (2009) Chinese Medicine Treatment of Adenomyosis Clinical Experience. Chinese Journal of Modern Drug Application, 2, 148.

[51] Ma, S.X. (2008) Clinical Observation of Modified Guizhi Fuling Wan Treating Endometriosis. Chinese Journal of Clinical Medical Research, 27, 9-11.

[52] Niu, W.G. (2007) Guizhi Fuling Wan Combined with Indometacin Capsules Treating 32 Cases of Pelvic Endometriosis. Journal of Practical Traditional Chinese Medicine, 23, 179.

[53] Li, R.G. (2007) Combined Traditional Chinese and Western Medicine Treatment of 50 Cases of Endometriosis. Liaoning Journal of Traditional Chinese Medicine, 34, 1127.

[54] Liu, Y.X. and Li, X.R. (2006) Traditional Chinese Integrated Methods Treating 31 Cases of Endometriosis. Journal of Clinical and Experimental Medicine, 5, 61.

[55] Qian, J., Zheng, L. and Ma, X. (2000) Clinical Research of Applying Method of Invigorating Kidney and Removing Blood Stasis to Treat Endometriosis. Journal of Nanjing University of Chinese Medicine, 16, 277-278.

[56] Dong, A.F. and Nei, Y. (2000) Prescription Combined with Guizhi Fuling Wan Treating 48 Cases of Endometriosis. Yunnan Journal of Traditional Chinese Medicine and Materia Medica, 21, 27.

[57] Zhang, Y.Y. and Yang, G.T. (1996) Modified Guizhi Fuling Wan Treating 38 Cases of Endometriosis. Shanghai Journal of Traditional Chinese Medicine, No. 6, 32.

[58] Jin, J.L. (1994) Modified Guizhi Fuling Wan Treating 95 Cases of Endometriosis. Liaoning Journal of Traditional Chinese Medicine, 21, 271-272.

[59] Luo, J.H. (1992) Modified Guizhi Fuling Wan Treating 50 Cases of Endometriosis. Hunan Journal of Traditional Chinese Medicine, No. 6, 30.

[60] Shen, Y.L. and Jin, J.L. (2006) Clinical Observation of Applying the Method of Invigorating Circulation of Blood and Removing the Stasis by Stages to Treat 27 Cases of Adenomyosis. Lishizhen Medicine and Materia Medica Research, 17, 2037-2038.

[61] Gao, H.M. (2002) Ectopic Pregnancy II Prescription and Guizhi Fuling Capsule Treating 30 Cases of Ovarian Endometriosis Cyst. Forum on Traditional Chinese Medicine, 17, 41.

[62] Li, M.Z. and Wang, C.X. (2007) Observation of Dahuang Zhechong Wan Combined with Gestrinone Treating 60 Cases of Endometriosis. Journal of Practical Traditional Chinese Medicine, 23, 641 . 
[63] Wei, L.J. (1998) Internal and External Treatment of Endometriosis. Jiangxi Journal of Traditional Chinese Medicine, 29, 24-25.

[64] Jin, T.F. and Li, M.J. (2005) Clinical Observation of Mifepristone and Dahuang Zhechong Capsule Treating Adenomyosis. The Chinese Modern Journal of Traditional Chinese and Western Medicine, 3, 36-37.

[65] Liu, X.F., Chen, L.S. and He, Z. (2004) Observation of 30 Cases of Dahuang Zhechong Wan Treating Adenomyosis. Journal of Practical Traditional Chinese Medicine, 20, 64-65.

[66] Fan, D.X. and Wang, L.M. (2004) Lei Gong Teng Pill and Dahuang Zhechong Wan Treating 88 Cases of Endometriosis. China's Naturopathy, 12, 45-46.

[67] Ji, Z.F. (2011) 80 Cases of Pelvic Endometriosis Clinical Curative Effect Observation of Different Treatments. Liaoning Journal of Traditional Chinese Medicine, No. 7, 3329-3330.

[68] Wang, Z. and Luo, A. (2009) Di Dang Decoction Treating 80 Cases of Endometriosis. Guangxi Journal of Traditional Chinese Medicine, 32, 19-20.

[69] Duan, Q.Z. and Jiang, X.P. (2012) Clinical Observation of Modified Yiyi Fuzi Baijiang Decoction and Di Dang Decoction Treating Endometriosis. Journal of Sichuan of Traditional Chinese Medicine, 30, 107-108.

[70] Zeng, J.B., Wang, T. and Xu, A.F. (2008) Clinical Analysis of Di Dang Decoction Treating Endometriosis. Journal of Liaoning University of Traditional Chinese Medicine, 10, 88.

[71] Wu, X.H. (2003) Di Dang Decoction Treating 58 Cases of Endometriosis. Journal of Emergency in Traditional Chinese Medicine, 44, 445.

[72] Zhang, Y.L., Wang, B.Q., Yue, Y., et al. (1998) Clinical Observation of Wen Jing Decoction Treating 45 Cases of Endometriosis. Chinese Journal of Traditional Medical Science and Technology, 5, 243-244.

[73] Zhen, H.P. (2012) Influence in Quality of Life in Patients with Endometriosis Dysmenorrhea of Wen Jing Decoction Treatment. Hebei Journal of Traditional Chinese Medicine, 34, 379-380.

[74] Chen, J.X. and Xu, H.X. (2003) Combined Traditional Chinese and Western Medicine Clinical Observation Preventing Recurrence of Endometriosis. Journal of Qiqihar Medical College, 24, 1370.

[75] Li, A.F. (2012) Clinical Observation on Treatment of Adenomyosis by Xia Yu Xue Decoction Combined Si Ni Powder. Chinese Journal of Information on Traditional Chinese Medicine, 19, 76-77.

[76] Zhu, Z.H. and Sun, R.R. (2001) Modified Xia Yu Xue Decoction Treating 42 Cases of Endometriosis. Journal of Sichuan of Traditional Chinese Medicine, 19, 49-50.

[77] Hou, Z.X. (2010) Modified Xia Yu Xue Decoction Treating 49 Cases of Endometriosis. Shanxi Journal of Traditional Chinese Medicine, 26, 19-20.

[78] Zhu, M. (2012) Clinical Observation of Treatment on 32 Cases of Adenomyosis Dysmenorrhea by Danggui Si Ni Plus Wuzhuyu Shengjiang Decoction. Beijing Journal of Traditional Chinese Medicine, 31, 916-918.

[79] Li, G.X. and Bian, F.F. (1997) Syndrome Differentiation Treatment on 20 Cases of Endometriosis Cyst. Correspondence of Traditional Chinese Medicine, 16, 16-17.

[80] Xue, Y.F., Lin, S.M. and Xu, W. (2006) ShenQiWan ShaoFuZhuYuWan and XiaoLeiWan match Clyster, Ion Ducting and Ear Acupuncture Method Treat Ectopic Endometrial Clinic Observe 500 Example. The Chinese Practical Journal of Traditional Chinese and Western Medicine, 19, 229-230.

[81] Liang, Q.X., Chen, H.R., Li, Z.M., et al. (2002) Huangqi Guizhi Wu Wu Decoction Treating 
34 Cases of Endometriosis. China's Naturopathy, 10, 54-55.

[82] Huang, X.R. and Qiu, X.P. (2007) Taohe Cheng Qi Decoction's Therapeutical Effect on Endometriosis. Clinical Journal of Traditional Chinese Medicine, 19, 231-232.

[83] Zheng, M.H. (2001) Experience on Danggui Shaoyao Powder Treating Endometriosis. Xinjiang Journal of Traditional Chinese Medicine, 19, 19-20.

[84] Wang, S.S. (2012) The Clinical Observation on the Treatment of Endometriosis with Modified Sini Decoction. Nanjing University of Chinese Medicine, Nanjing.

[85] Cheng, H.L. and Li, J. (2009) Clinical Curative Effect Evaluation of Modified Si Ni Powder Treating Endometriosis Dysmenorrhea. Liaoning Journal of Traditional Chinese Medicine, 36, 1324-1326.

Submit or recommend next manuscript to SCIRP and we will provide best service for you:

Accepting pre-submission inquiries through Email, Facebook, LinkedIn, Twitter, etc.

A wide selection of journals (inclusive of 9 subjects, more than 200 journals)

Providing 24-hour high-quality service

User-friendly online submission system

Fair and swift peer-review system

Efficient typesetting and proofreading procedure

Display of the result of downloads and visits, as well as the number of cited articles

Maximum dissemination of your research work

Submit your manuscript at: http://papersubmission.scirp.org/

Or contact ojog@scirp.org 